Review Article

Open Access

\title{
Mainspring of the Universe -The Mechanism Sustaining the Universe's Steady State
}

Conrad Ranzan

DSSU Research, Canada

\section{Article Info}

*Corresponding author:
Conrad Ranzan
DSSU Research
5145 Second Avenue
Niagara Falls, L2E 4J8
Canada
E-mail: DSSUresearch4@aol.com

Received: November 27, 2021

Accepted: December 06, 2021

Published: December 14, 2021

Citation: Ranzan C. Mainspring of the Universe -The Mechanism Sustaining the Universe's Steady State. Int J Cosmol Astron Astrophys. 2021; 3(1): 145-157. doi: 10.18689/ijcaa-1000128

Copyright: @ 2021 The Author(s). This work is licensed under a Creative Commons Attribution 4.0 International License, which permits unrestricted use, distribution, and reproduction in any medium, provided the original work is properly cited.

Published by Madridge Publishers

\begin{abstract}
Presented is the remarkable story of how the energy of the Universe is continuously replenished. The elements of the mechanism were developed over the past 20 years, have now been assembled together into what is being called a Mainspring object and is being presented here for the first time. From an unprecedented and unique perspective, the article delves into the Mainspring object (somewhat analogous to a nonsingular black hole) and its four functional components: (1) The key component is the Blueshifting mechanism. Applicable to the Mainspring's surface-trapped photons and neutrinos, this is the process of energy generation by velocity differential propagation of radiation. (2) The conversion component. Infalling mass transforms to pure energy. (3) The Primary radiation component. It is the ejection of amplified energy and the energy derived from mass infall. (4) The heretofore unrecognized component. Following logically from the defined principle of physicality, any excess mass acquired by the Mainspring object leads to mass extinction at the core - a process of mass destruction by aether deprivation.

The operation of the Mainspring object presents science with a supreme advantage. Unlike the situation plaguing $20^{\text {th }}$-century black-hole theory, nowhere is there a breakdown of the underlying physics. Moreover, it opens the door to a cosmology with no initial-conditions problem, no end-of-the-universe predicament, and no ultimatesource-of-energy mystery.
\end{abstract}

Keywords: Cosmology; Mainspring processes; Terminal star; Energy amplification; Blueshifting mechanism; Mass Extinction; Mass Annihilation; Aether Deprivation; Black hole physics; DSSU

\section{Introduction}

In the 1930s, physicist and philosopher Sir James Jeans expressed concern over the fact that "the solid substance of the material universe is continually dissolving away into intangible radiation." He cited the example of the Sun, which, every 24 hours converts 360,000 million tons of its material into radiation - radial emission that is, for the most part, "destined to journey on through space until the end of time." Jeans pointed out, the same conversion of mass to radiant energy is in progress in all the stars of the universe. He then wondered whether there was some way of circumventing such ongoing disintegration of the material world:

"It is natural to ask whether a study of the universe as a whole reveals these processes as part only of a closed cycle, so that the wastage which we see in progress in the sun and stars and on the earth is made good elsewhere. When we stand on the banks of a river and watch its current ever carrying water out to sea, we know that this water is in due course transformed into clouds and rain which replenish the river. Is the physical universe a similar cyclic system, or ought it rather to be compared to a stream which, having no source of replenishment, must cease flowing after it has spent itself?" -Sir James Jeans [1, p343] 
Jeans understood that the radiated energy cannot run downhill forever like the flow of a rain-replenished river, rather, like the weight driving a pendulum clock, it must ultimately touch bottom.

"The energy is still there, but it has lost all capacity for change; it is as little able to work the universe as the water in a flat pond is able to turn a water-wheel. [... And so] the universe as a whole cannot so go round and round."[1, p347]

Along with his contemporaries, James Jeans believed the universe had no extraneous font of energy. He thus forecast a cosmic lack-of-heat death:

"[T]he energy of the universe must continually lose availability; [ultimately] a universe in which the energy had no further availability to lose would be dead already. Change can occur only in the one direction, which leads to the heat-death. With universes as with mortals, the only possible life is progress to the grave."[1, p347-348]

Contrary to James Jeans' assessment and similar predictions associated with $20^{\text {th }}$-century cosmology, it turns out that the universe does have a "source of replenishment." It is now possible to scientifically address and resolve the fate-of-the-universe problem.

The source of replenishment is an object best described as an end-state neutron star. Its theoretical foundation is directly linked to the aether theory of gravity [2] and three recently discovered laws of physics:

- The Velocity Differential propagation of light. [3]

- Energy Generation via Velocity Differential Blueshift. [4]

- Mass Extinction by Aether Deprivation process. [5]

The functionality and importance of these laws will be examined in the course of the discussion. But first, I want to give an overview of the end-state neutron star, which, for brevity and convenience, will be called the Terminal star, and, in recognition of its key importance, designated the Mainspring object.

\section{Terminal-State Star}

The Terminal-state star is essentially a neutron star that has acquired a lightspeed surface-boundary, which means the space-medium inflow at the 'surface' equals the maximum allowable. Ontologically, it is an end stage object; its state of being cannot be altered in any way other than changes of rotation. Such an object is truly in an end state of existence. It is both a destroyer of energy (specifically, mass energy) and a generator of energy (specifically, it amplifies the energy of photons and neutrinos). The Terminal star's defining feature is its unique surface; the star is enveloped by an energy surface/layer onto which the space medium (aether) flows at the speed of light. Moreover, this energy layer encloses a fixed quantity of mass existing as nature's ultimate density state. If the density is assumed to be the same as nucleons (about $1.6 \times 10^{18} \mathrm{~kg} / \mathrm{m}^{3}$ ), then the radius of the Terminal star must be about 10 kilometers. Another unique feature is its manner of rotation. When rotating, it manifests no centrifugal effect [6]. The only restraint on the rate of rotation is the resistance from the magnetic lines of force. In the absence of a magnetic field, there would be no theoretical spin limit and, of course, no way of measuring it. The Terminal star's most important external feature is its pair of emission beams.

The anatomy of the Terminal star, or end-state neutron star, is illustrated in Figure 1.

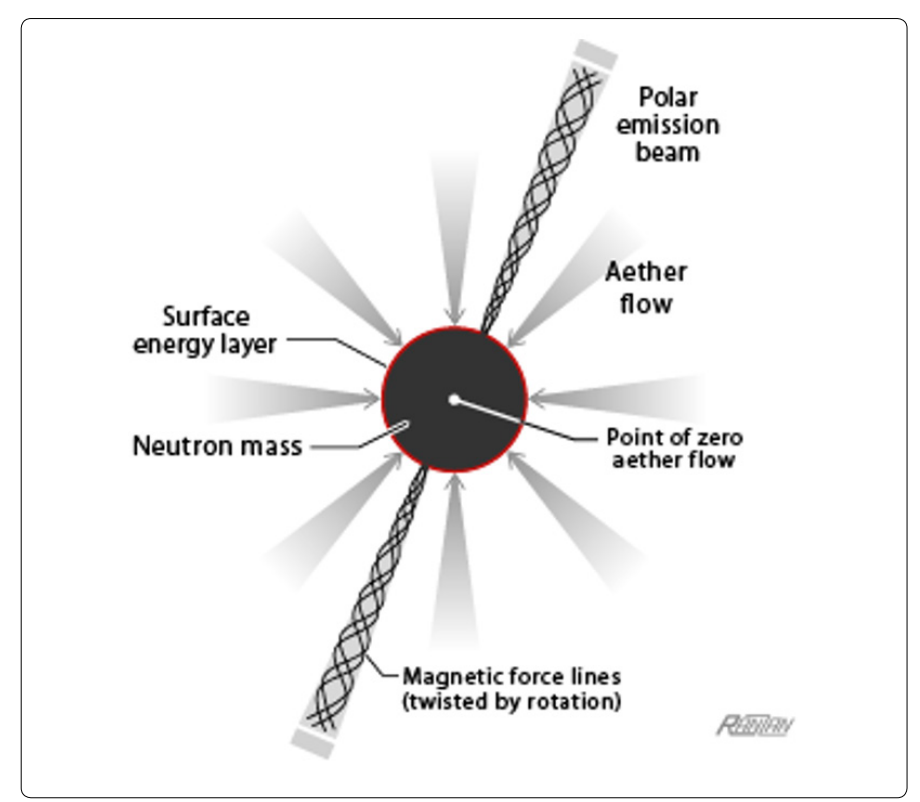

Figure 1. Anatomy of the end-state neutron star (shown in cross-section). This object is an "end-state" star -or Terminal star- in the sense that it cannot be altered in any way whatsoever, other than changes of rotation. All such stars, by virtue of elementary governing laws of physics, are exactly identical (identical, except, of course, for the spin rate). Based on the neutron mass having a density of $1.6 \times 10^{18} \mathrm{~kg} / \mathrm{m}^{3}$, the star has a radius of about 10 kilometers. This unique type of star serves as the universe's Mainspring object.

Theoretically predicted and observationally confirmed, this is without question the universe's most unusual type of star. Once such a star forms, it can neither grow larger nor smaller. Its physical volume and mass content remain forever fixed [5].

\section{So, how does such a star form?}

The formation of Terminal neutron stars is based on the highly-reasonable assumption that mass cannot be compressed beyond some specific density. This maximum density is generally acknowledged to be the density of atomic nuclear particles, such as the neutron. When gravitational collapse, or gravitational aggregation, brings about the ultimate density state of matter and also attains a contiguous quantity limit as determined by the special theory of relativity, the resulting object is a Terminal neutron star. Restated, the said transformation requires the satisfaction of two conditions: (i) The maximum density that nature allows; and (ii) the maximum space-medium inflow that nature allows (as referenced to the surface of the collapsed object). In conventional black-hole physics, this latter condition is sometimes stated in terms of the gravitating entity having an escape speed (with respect to its event horizon 'surface') equal to the speed of light. 
Important information about the space medium. Be aware that the space medium plays an essential role in the Universe. It not only facilitates the propagation of electromagnetic radiation [7], but also functions as the veritable cause of gravity - something missing in both the Newtonian forcetype gravity and the Einsteinian geometric-type gravity. It is often called the vacuum, or the quantum foam, or the fabric of the cosmos; but, in its role within cellular-cosmology theory (DSSU), it is simply called aether. This universal aether medium, as is now known, has truly unique properties. The Terminal star/Mainspring functions as it does precisely because the universal substrate has been recognized as being nonmaterial and discretized (and having the dynamic ability to maintain a constant-count density).

Additional details of the process of the collapse to the Terminal state may be found in reference [8]. And for details on the DSSU aether theory of gravity see the very readable article, The Nature of Gravity -How one factor unifies gravity's convergent, divergent, vortex, and wave effects [2].

\section{Mainspring Mechanism}

The Mainspring mechanism can be divided into four components. Three of these are involved in the energy generating function.

(1) Matter input. The capture of 'low energy' matter, including microwave background radiation, background neutrino radiation, and ordinary mass particles. Also, significant mass objects may be captured. In a binary system scenario, the 'input' may manifest as the gradual cannibalizing of a gaseous partner.

(2) Energy amplification. A remarkable process that follows directly from a recently discovered mechanism, the velocity differential propagation of light. Energy is gained by the elementary process of spectral Blueshifting.

(3) Polar emission of radiant energy. This is where reenergized photons and neutrinos, in a broad range of energies and intensities, escape to the outside world. Poetically, this is the Universe's perpetual fountain of youth.

(4) Terminal Extinction of Mass. The fourth component acts as a regulator on the internal mass content of the Terminal star.

A schematic of the various components is shown in Figure 2.

It should be emphasized, the physics underlying the entire Mainspring system is surprisingly straightforward. For one thing, it adheres to the parsimonious dictum that the proper goal of physics is to make as few underlying basic assumptions as possible to explain as broad a spectrum of phenomena as possible [9]. In fact, only two fundamental assumptions, applicable to the physical realm, are required. One assumption, as already pointed out, is that gross mass has a limited degree of compressibility. No amount of gravitational compression can exceed that natural density limit.
The other assumption is that matter (both mass and energy particles) absorbs/consumes the universal medium (aether). Matter acts as the sink, aether as the source. This relationship between matter and aether reaches to the deepest ontological level. Essentially, matter exists as a consumptive excitation of aether. Simply put, mass exists only as long as it is supplied with aether. It is the core idea behind the aether theory of gravity. And of immediate importance, this existence-sustaining relationship is the key to the operation and understanding of the fourth component mass extinction by aether deprivation.

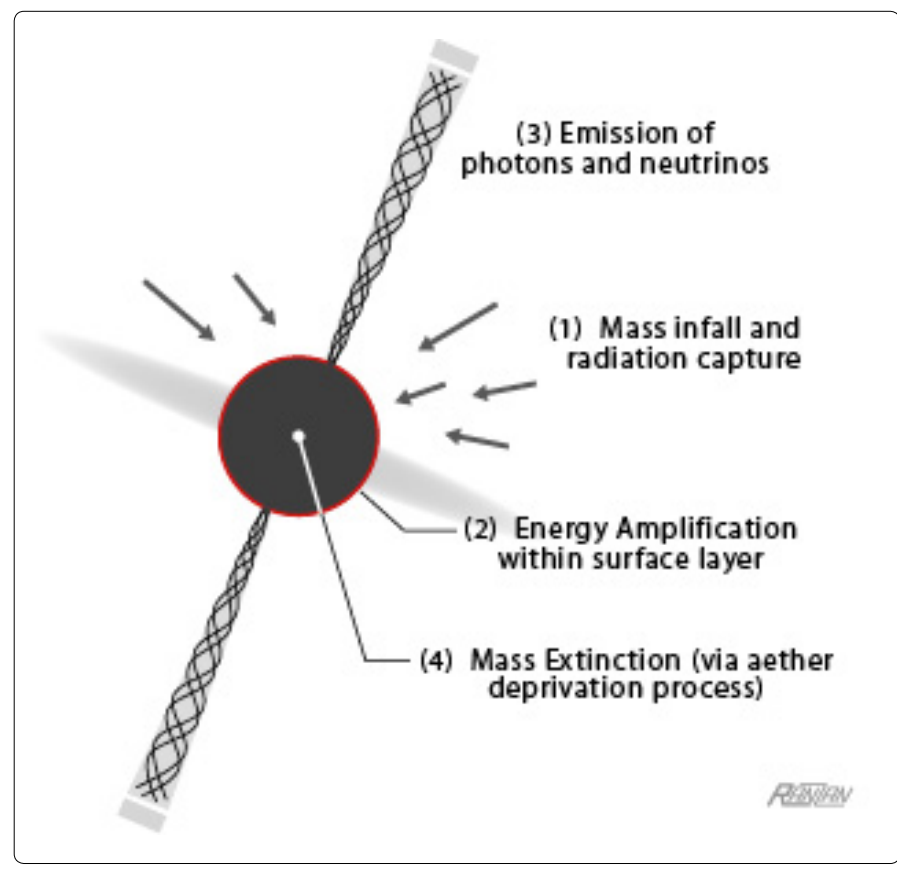

Figure 2. Mainspring object's role as an energy generator involves three essential components or functions: (1) Matter input;

(2) Blueshift amplification of surface-trapped photons and neutrinos; (3) Radiant energy emission through opposite poles.

Separate from the energy-producing function, the Terminal neutron star (shown in cross-section) also has a component (4) for the elimination of any excess mass.

\subsection{Matter Input}

The Terminal star's extremely powerful gravitational effect 'pulls' in everything within reach. Gas, dust, space rocks, along with all kinds of radiation are dragged in. Background radiations —starlight, microwave photons, and ubiquitous neutrinos - pour in from all directions. If an accretion disk is present, its material gradually spirals inward in accordance with the aether theory of gravity. If the Terminal star is part of a binary system, the mass inflow may involve the gradual consumption of a gaseous partner.

The infall of routine mass (what in a terrestrial environment would be classified as meters) causes disturbances in the energy surface that spread as miniature tidal waves racing over the entire spherical envelope, prompting bursts of polar emissions. But what happens when the mass infall is of a more significant nature? There is also an extreme scenario to consider. What happens when two Terminal stars undergo a gravitational merger? ... These situations are discussed in Section 3.4. 


\subsection{Blueshift Amplification}

The beating heart of the energy generating system is the thin surface layer of the Mainspring object. That is where trapped photons and neutrinos undergo a relentless process of Blueshifting. The process is the result of these particles propagating solely within this layer — where a small radial velocity differential (a velocity differential of the inflowing aether) causes propagating waves to contract over time. The contraction of their wavelengths means the photons and neutrinos are being blueshifted. The contraction of their wavelengths means they are gaining energy! Hence, the process is referred to as Blueshift amplification.

It is a most remarkable process and follows directly from the recently discovered mechanism of the velocity differential propagation of light. Normally, the velocity differential mechanism leads to a loss of energy (as occurs in the cosmic redshift), but under the unique conditions present within the Terminal star's surface, it works as an energy gain. Energy is gained by the elementary process of spectral Blueshifting.

While trapped and propagating 'in-place' in the surface layer, photons and neutrinos undergo a gradual, but relentless, wavelength contraction. The proof of this contraction is surprisingly simple.

Consider a representative photon trapped in the surface, as shown in Figure 3. This photon is moving outward at lightspeed, while the aether is flowing inward at lightspeed; the result is a 'stationary' photon (a photon just propagating in place). But the aether flow is not constant; rather, it changes with respect to the radial distance. In accordance with the aether gravity theory, the magnitude of the inflow velocity (of aether) varies as indicated by the graph. Clearly there exists an inflow velocity difference at the photon's location. The photon 'experiences' a velocity difference between its two ends. When analyzed, it is found that the front and back ends are actually moving closer together [4].

Given that the photon is an extended entity and always travels at speed $c$ with respect to the aether medium, the following must be true:

(Relative velocity between ends of photon $)=($ vel of front end) - (vel of back end),

$$
\begin{aligned}
& =\left(c+v_{1}\right)-\left(c+v_{2}\right), \\
& =c+v_{1}-c-v_{2}, \\
& =\left(v_{1}-v_{2}\right)<0 .
\end{aligned}
$$

Note that aether flows $v_{1}$ and $v_{2}$ are both negative (i.e., inward to the structure). But because $v_{1}$ is more negative than $v_{2^{\prime}}$ the bracketed expression (2) must be negative indicating a converging situation.

Consequently, any surface-embedded photons and neutrinos undergo energy amplification - they slowly gain energy.

Additional elements of the proof of this energy-gaining spectral shift are presented in reference [4]. (Details of the opposite effect - the velocity differential Redshift- are presented in references [3] and [10].)

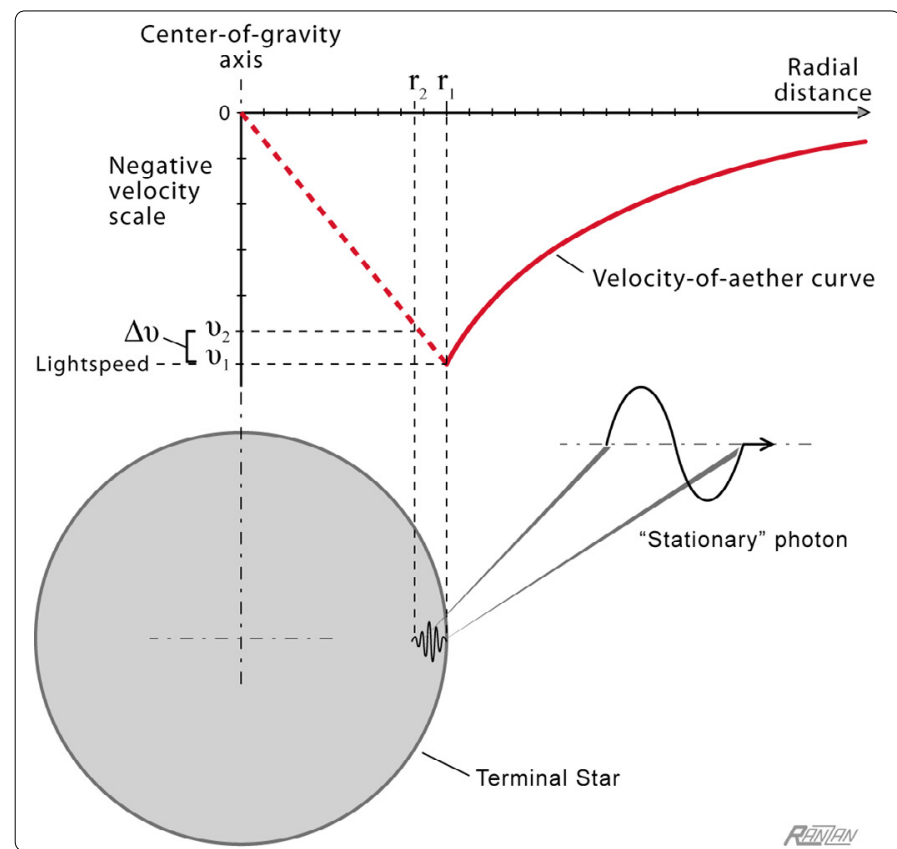

Figure 3. Energy particle amplification. The schematic reveals the cause of wavelength contraction of any surface-embedded photon. By virtue of propagating within a zone of decelerating aether, the surface photon undergoes continuous blueshifting. Essentially, it gains energy. The photon is being conducted by a space medium whose speed of inflow decreases. As a result, the front and back

ends of the photon 'experience' a flow differential. It is the

algebraic value of this differential that proves (see text) the embedded photon undergoes contraction. (The "velocity-of-aether" curve is derived from the aether theory of gravity. The dashed curve approximates the interior aether-flow function. Photon size is, of course, greatly exaggerated.)

Another energy process occurs in the surface layer. It is an instantaneous conversion of some of the infalling mass into pure energy -into photonic and neutrino forms of energy.

Consider an incoming electron (or nucleon) striking the surface. It can't bounce off the super-dense energy layer. It cannot because doing so would require the "bounced" particle to travel faster than the speed of light in vacuum just not possible. Remember, the aether space medium is flowing into the surface layer at full-value lightspeed.

Moreover, the impacting particle (regardless of its kinetic energy) cannot join with the ultra-dense mass just beneath the energy layer. The reason has to do with the original premise. The assumption was that this mass exists in a state of maximum density - the maximum that nature allows. That density cannot be increased and neither can the total mass.

Only one option is available. The particle becomes trapped in the energy layer; and for that to happen, it must transform into pure radiant energy.

When, however, too much mass suddenly falls in, things get interesting.

When large mass objects strike the Terminal star, some of the mass will be converted to energy as just described. The rest will join the subsurface main-body mass, in spite of the above restrictive remarks. Nonetheless, such additional mass does not increase the density and does not alter the total mass. The explanation is given in Section 3.4 below. 


\subsection{Radiant Energy Emission}

The photons and neutrinos trapped within the Terminal star's energy layer, as may be imagined, carry a wide range of energies. As long as they remain trapped, their energy will progressively increase. The only way to escape is for the particles to reach a polar opening. It is there that the tightlyconstricted magnetic field maintains openings through the lightspeed barrier. Actually those magnetic force lines prevented the lightspeed barrier from forming there in the first place. By the very act of consuming aether, the concentrated magnetic energy (which is extremely concentrated along the polar axis) reduces the flow of incoming aether thereby preventing the inflow from attaining the full speed of light. Thus, the Terminal star's ultimate barrier between inside and outside has its weak points -its two escape portals.

The mechanism by which trapped radiation reaches the portals can be surmised. The Terminal star's energy layer has extraordinary density; so there is a powerful lateral pressure. This pressure continuously feeds the polar emission beams (Figures 1 and 4). Also, any impacting 'chunks' of mass will generate reverberations of the structure -low amplitude but fast Tsunami-like waves. Any such disturbances of the Terminal star's surface will produce bursts of energy emissions which superimpose on the normal emission level.

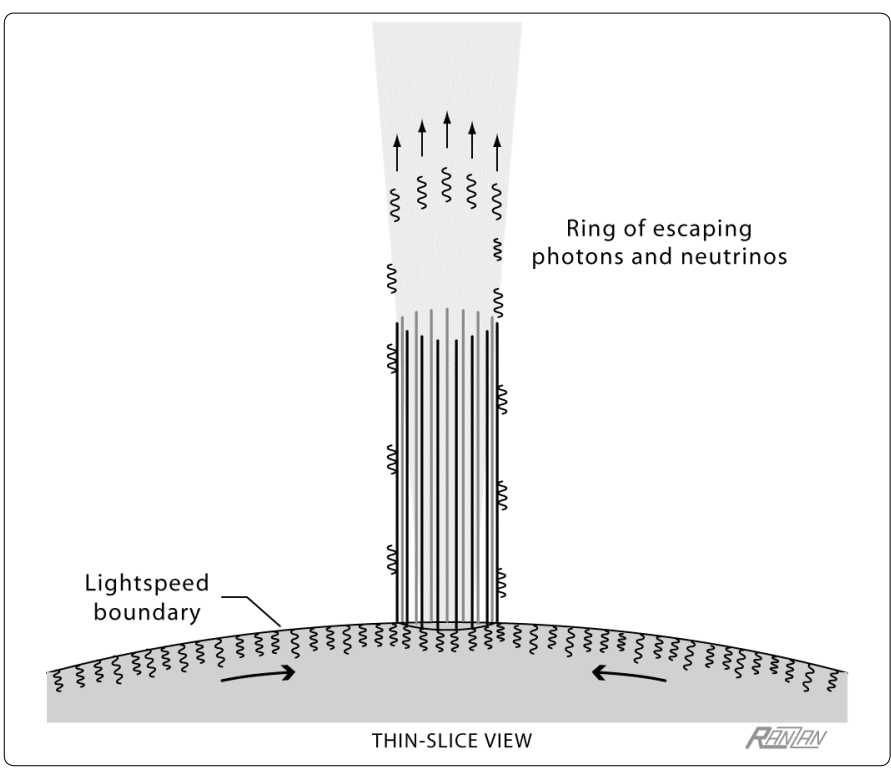

Figure 4. Energy emission mechanism of Terminal stars. Energy particles (photons and neutrinos) make their escape through the polar portal and outward along the magnetic channel. The extreme

density of the energy layer produces a lateral pressure that continuously pushes surface-embedded particles towards the portals where, because of the greatly reduced aether 'headwind' there, they are able to escape. Elsewhere the radiation is prevented from escaping by an aether lightspeed headwind.

There is considerable variability in the energy level of the ejecting particles at the point of escape. But immediately upon release another cause of variation comes into play. It has to do with the intensity of the magnetic field through which the released radiation must propagate. Several factors are tied-in here: the magnetic field energy density, the rate of rotation, aether consumption, and the resulting aether inflow speed. The energy density of the magnetic field depends on the degree of collimation, which, in turn, depends on the rate of rotation of the neutron star. Then, the combination of the magnetic energy density and ejecta energy determine the quantity of aether consumption which in turn affects the local speed of aether inflow (in the environ of the magnetic column and the ejecta cylinder). Finally, it is the aether velocity (namely, its derivative) that determines the redshift (the velocity differential redshift) that the outbound radiation suffers. All emitted radiation will have its energy reduced to some degree, or other, during outbound propagation. This is a natural occurrence -a consequence of a velocity differential redshift.

The end result is the broadcasting of a wide assortment of radiation energies: From radio waves, through microwaves and visible light, to $x$-rays and gamma photons; from garden variety neutrinos to ultra-high energy PeV neutrinos and higher.

If it were somehow possible to observe the emission close-up at a polar opening, the image would be a curtain of 'light' around the opening - a cylindrical shaft of highintensity energy blasting into the external environment of deep space.

This concludes the examination of the three components of the energy generating feature of the Mainspring object. What follows next is the explanation of size uniformity - the workings of the mechanism by which Terminal stars maintain a fixed total mass content.

\subsection{Terminal Extinction of Mass}

The best way to explain this process, and why it is necessary, is to examine what would happen if a significant quantity of mass abruptly joins with the Terminal star. Assume, just for a moment, that no mass extinction occurs. If, as mentioned earlier, the maximum density is $1.6 \times 10^{18} \mathrm{~kg} / \mathrm{m}^{3}$, then the radius of the Terminal star will be about 10 kilometers and its total mass must be 3.4 solar masses.

Let us say the mass being added is equivalent to one solar mass. The combined mass, the total mass, will now be $4.4 M_{\odot}$. Since the density does not change (it cannot change because of the original assumption), the star's radius must increase to accommodate the increase in mass. A simple calculation gives the new radius of 10.93 kilometers. ${ }^{1}$

In order to sustain the existence of the $4.4 M_{\odot}$ (at neutron density) inside a 22-kilometer-diameter sphere, the aether inflow at the surface would have to be $3.27 \times 10^{8} \mathrm{~m} / \mathrm{s}^{2}$

But there is an obvious problem here: The inflow speed just calculated is 9 percent above the speed of light - a clear violation of special relativity. The calculation is saying the structure has too much mass, or equivalently, it has too much

\footnotetext{
1 Radius of sphere (as a function of density): $R(\rho)=\left(\frac{3}{4 \pi} \frac{M}{\rho}\right)^{1 / 3}$, where $\rho$, the density, may be uniform or average. With mass $M=4.4\left(1.99 \times 10^{30} \mathrm{~kg}\right)$ and density $\rho=1.6 \times 10^{18} \mathrm{~kg} / \mathrm{m}^{3}$, the expression gives the radius as $10.93 \mathrm{~km}$.

2 In accordance with the aether theory of gravity, the speed of aether inflow at the surface radius $R$ of mass $M$ is $v_{\text {inflow }}=\sqrt{2 G M / R_{\text {surface }}}$, where $G=$ $6.673 \times 10^{-11} \mathrm{~N} \cdot \mathrm{m}^{2} / \mathrm{kg}^{2}$. With $M=4.4\left(1.99 \times 10^{30} \mathrm{~kg}\right)$ and $R_{\text {surface }}=10.9 \times 10^{3} \mathrm{~m}$; $\mathrm{u}_{\text {inflow }}=3.27 \times 10^{8} \mathrm{~m} / \mathrm{s}$.
} 
gravity. And increasing the density in order to make the structure smaller won't help; it would only make the special relativity violation worse; the surface inflow would become still faster.

Moreover, increasing the size of the sphere in order to enlarge the surface area for aether to flow through won't work either. The mass body is already in the Terminal collapsed state - there is no force to counter it, nothing that can overcome it.

There is only one option, and that is to end the existence of the excess mass.

This is exactly what happens. However, it is not the added mass that undergoes extinction; rather, it is the mass at the core of the structure that ceases to exist. It is the core mass that suffers aether deprivation. It makes perfect sense. Surface mass is supplied with aether first and if the supply runs out before it reaches the core, then the core mass simply can no longer exist. See Figure $\mathbf{5}$.

Thus, the $4.4 M_{\odot}$ temporary structure would lose 1.0 solar mass by the process of aether deprivation — and it will do so within a fraction of a second.

The quantity of contiguous end-state mass can never be changed!

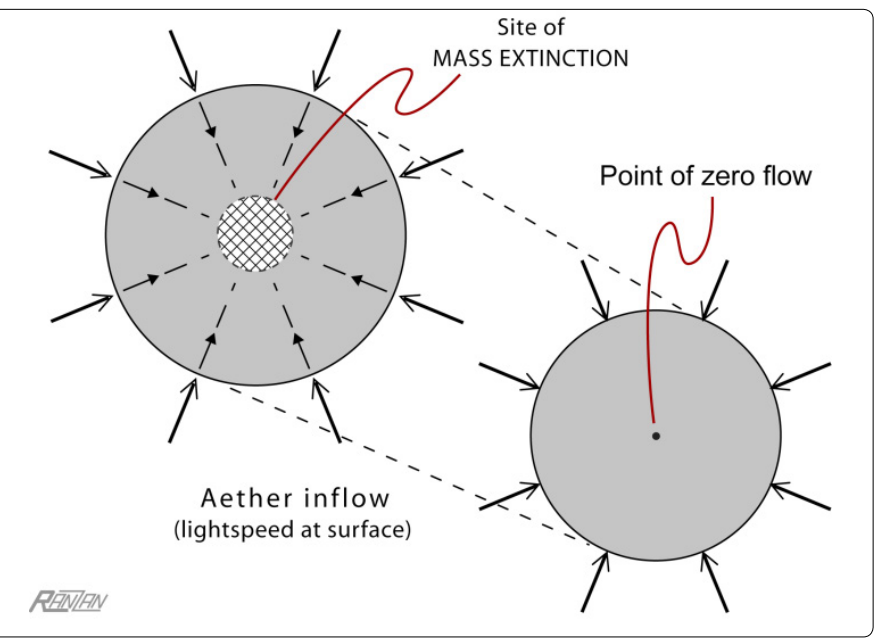

Figure 5. Mass extinction by Aether Deprivation. If the aether is consumed before reaching the center-of-mass of the structure, then the core region (crosshatched) suffers aether deprivation. The mass therein will instantly cease to exist. The surrounding mass will immediately take the place of the extinguished mass, thereby preventing any pocket of nothingness from actually forming. During the extinction process, the structure correspondingly contracts to the 20-km-diameter (approximately) stable size of all end-state neutron stars (as represented by the right-hand image).

In the normal course of the existence of a Terminal star, the process of mass extinction is a very low level activity and not something detectable. For a structure experiencing significantly greater activity, it seems reasonable to assume that whenever there are bursts of energy emission (as indicated by the observable effects on so-called astrophysical jets) there are accompanying episodes of mass extinction at the core (but hidden from view). An active Terminal star is typically one that is absorbing material from its orbiting gaseous partner.
The process, at times, performs on an unparalleled level -an astonishing degree of mass loss. The most dramatic extinguishment of mass occurs when two Terminal stars merge (Figure 6). Such a merger, by way of a spiral orbital engagement or a rare collision, represents the ultimate instance of the principle of invariance of contiguous end-state mass. When one Terminal star merges with another, the result is exactly one Terminal star. Other than some above-normal bursts through the emission beams, there is absolutely no byproduct. The merger event (an almost instantaneous melding) marks the total vanishment of ultra-dense mass, equivalent to about 3.4 solar masses [5].

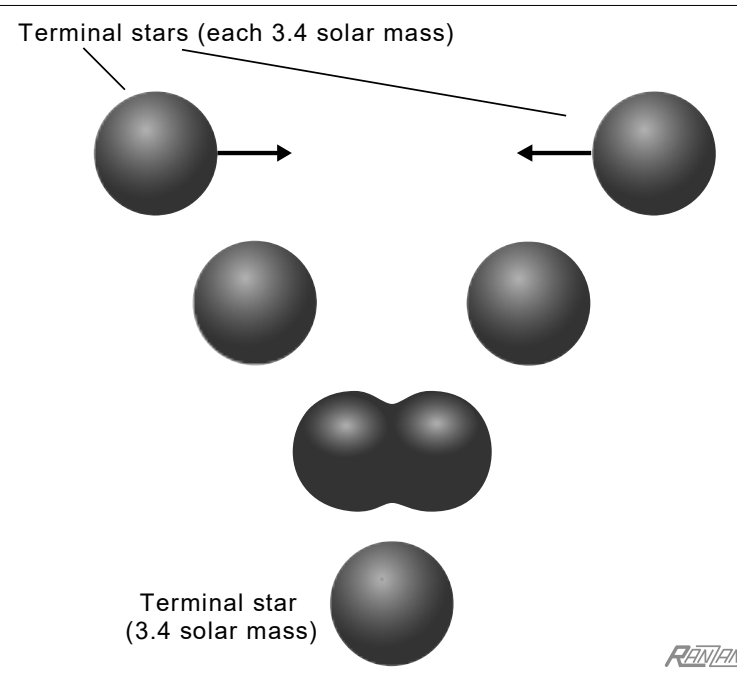

Figure 6. Mass extinction on a spectacular scale occurs when Terminal stars merge. The merger may come about through an orbital engagement or an outright collision (as shown here). The end result is a single Terminal star identical in size and mass to just one of the original. Only the rotation rate (and the orientation) is subject to change. Remarkably, the mass equivalence of 3.4 Suns completely vanishes. (Polar jets are usually present, but are not shown.)

Importance of the fourth component. The extinction-ofmass mechanism serves the immediate purpose of maintaining the Terminal star's mass load. But there is a deeper purpose. In the larger context, this mechanism (always of course acting within Terminal stars) prevents the greater universe from accumulating excessive mass-energy. It is the disposer of mass overabundance. This fourth component is the overflow valve in the perpetual production/formation of mass from the perpetual streams of radiation emanating from the multitude of Terminal stars.

The extinction mechanism is the Mainspring acting as its own governor/controller -acting as the ultimate destroyer of the fruits of its own production.

\section{Formation of Matter and Production of More Mainspring Objects}

To the outside world the Mainspring objects are a source of gravity and, most importantly, the ultimate source of radiation - namely photons and neutrinos. Emanating from polar emission beams, these energy particles are broadcast haphazardly throughout the universe. So, what happens to all this expelled energy? First of all, we can be sure of one thing: 
The particles/waves travel the universe until there is a collision of one kind or other. Sooner or later there will always be a collision - an interaction, a reflection, a capture, or whatever.

\subsection{Kinetic Energy Transfer}

Photons and neutrinos are carriers of momentum $\mathbf{p}$. The momentum of photons is usually expressed in terms of energy. The justification works like this:

A photon carries energy $E=h f$;

Energy, naturally, has an equivalence to mass, as in $E=m c^{2}$;

The photon then has mass equivalence $m_{v}=\mathrm{E} / \mathrm{c}^{2}=\mathrm{hf} / \mathrm{c}^{2}$;

And since it is traveling at lightspeed, it has a corresponding momentum $\left|\mathbf{p}_{\gamma}\right|=m_{\gamma} c=\left(h f / c^{2}\right) / c$;

which simplifies to $\left|\mathbf{p}_{\gamma}\right|=\mathrm{hf} / \mathrm{c}_{\text {; }}$ where $\gamma$ is the symbol for the photon, $h$ is Planck's constant, $f$ is the photon's frequency, and $c$ is the lightspeed constant.

Expressed in terms of energy,

Momentum-Energy $=\left|\mathbf{p}_{\gamma}\right| c=h f$.

The reason this is so important is that momentum can be transferred. Whenever the Mainspring-sourced particles encounter and interact with mass particles, regardless of whatever else happens, there will be a transfer of momentum energy, resulting in a gain or alteration of kinetic energy of the struck particle. It is this transfer of momentum energy that drives astrophysical jets by propelling the constituent mass particles (electrons and nucleons) to relativistic speeds.

Herein lies the explanation of ultra-high-speed particles that have repeatedly been detected when studying cosmic rays. Individual cosmic ray particles, high-speed nuclei and electrons, have energies ranging "from about $10^{6}$ to more than $10^{20} \mathrm{eV}$."[11] Understand what this means, try to grasp the enormity of the momentum being conveyed. A simple hydrogen nucleus consists of a proton. The greatest energy that the most powerful particle accelerator on Earth can bestow on a proton is about $7 \mathrm{TeV}$ (that's $7 \times 10^{12}$ electron volts). Compared to this $7 \mathrm{TeV}$, the particle's own mass energy is negligible. Almost all of the energy, then, is in the form of the energy of motion (momentum energy and kinetic energy). The existence of ultra-energy particles ranging from $10^{15} \mathrm{eV}$ to more than $10^{20} \mathrm{eV}$ is quite simply mind boggling. How in the world does one explain protons and nuclei with this kind of momentum/energy!? There is no known mechanism - no collision, no reaction, no process, no theoretical constructthat can even come close to imparting energy of this level, except one. The energy amplifying mechanism of the Mainspring object is the only possible way to load up photons (and neutrinos) within this magnitude range - with such enormous potency - which those photons can then transfer to mass particles as momentum-energy.

Incidentally, this transfer-of-momentum mechanism is the means by which ordinary stars manage to expel dust particles (and dust clouds) from their surrounding space. Photon bombardment is the means by which stars prevent being enshrouded by interstellar particles and becoming lost in the fog, so to speak.

\section{2. 'Starlight' Augmentation}

As sort of a general phenomenon, Mainspring-sourced radiation tends to boost the energy available for stars to carry on their thermal activity.

Energy particles that strike ordinary stars represent an addition to the energy available for re-emission. They augment the existing thermal energy, and probably prolong the lifespans of stars.

\subsection{Mass/Matter Formation}

The formation of matter requires only one thing - energy. The energy may be in the form of kinetic mass or in the form of radiation. There are basically three ways to generate or 'create' more mass: (1) high speed mass-on-mass collisions; (2) collisions between high-energy radiation particles; and (3) high-energy radiation colliding with mass particles.

A closer look at the first way. When experimental physicists collide mass onto mass, they end up with more mass than they started with. The giant particle accelerators like those at CERN, Fermi lab, Brookhaven, and elsewhere do this routinely.

Consider these classic examples from the CERN laboratory near Geneva. Within the Large Electron-Positron Collider, which operated during the 1990s, electrons and positrons (antielectrons) were accelerated to velocities within about one part in a hundred billionth $\left(10^{-11}\right)$ of the speed of light. Speeding around in opposite directions, the particles smashed into each other and produced a lot of debris. A typical collision might produce ten PI mesons, a proton, and an antiproton. Here's a comparison of the total masses, before and after:

$$
\text { Electron + positron: } 2 \times 10^{-28} \mathrm{gram}
$$

Ten pions + proton + antiproton: $6 \times 10^{-24} \mathrm{gram}$

Remarkably, what comes out weighs about thirty thousand times as much as what went in [12, p16]. The electron and positron in the reaction converted their prodigious kinetic energy into more mass - amounting to thirty thousand times their rest mass!

Mass 'creation' also occurs with protons. As particle physicist Frank Wilczek (Nobelist, Physics, 2004) noted, "If you bang protons together really hard, what you find coming out is ... more protons, sometimes accompanied by their hadronic relatives. A typical outcome would be, you collide two protons at high energy, and out come three protons, an antineutron, and several $\mathrm{PI}$ mesons. The total mass of the particles that come out is more than what went in."[12, p31]

The conclusion, in the context of cosmic rays, is this: the greater the kinetic energy of an incident mass particle, the greater will be the mass production upon impact (wherever and whenever it may occur).

The second way to 'create' mass. The interaction (collision) between two massless particles can also produce mass. For example, two photons with sufficient energy and under the right conditions will produce an electron-positron pair.

The third way to 'create' mass. Under this category massless particles collide with mass particles. Occasionally a neutrino will hit a proton and convert it into a positron plus a neutron (as reported in Discover magazine, August 1988, p21). These 
events are indirectly observable because the electrically charged positron tears off at great speed, emitting photons visible as Cherenkov radiation within the medium of the particle detector. The Cherenkov radiation is a cone-shaped beam of light that precedes the positron along its path. Since a neutron weighs more than a proton and in addition a positron has been produced, the collision clearly has generated new mass. The post collision mass is greater.

Among the most spectacular examples of nature's ability to produce mass from pure radiation are associated with the IceCube neutrino observatory located in Antarctica. Neutrinos are the most abundant particles in the universe; there are a billion $\left(10^{9}\right)$ of them for every existing atom $[13, \mathrm{p} 9]$. Although Earth receives a constant flux from deep space, the neutrinos are highly elusive. Hence, special large detectors are necessary for the study of the rare interaction events that do occur. The relevant point here is that when a rare collision happens, the energy of the incident neutrino is determined by the quantity of mass (the subatomic particles) produced and described as a shower of charged particles that radiate blue light (Cherenkov radiation).

The IceCube observatory has recorded events from neutrinos having energy more than a thousand times higher than any neutrinos that have ever been produced in particle accelerators on Earth "and almost a billion times the energy of the neutrinos regularly spat out by the sun."[14] They were dubbed "PeV events" because their energy fell in the petaelectron-volt $\left(10^{15} \mathrm{eV}\right)$ range. Putting this energy in perspective, it corresponds to about a million times the mass energy of a proton! [13, p22] Imagine one massless neutrino with the equivalent energy of $1,000,000$ protons.

Physicists understand that neutrinos are created during beta decay of an atomic nucleus, resulting in the release of an electron and the newly minted neutrino. They understand that the neutrino does not reside in the nucleus to begin with, rather, it is created during the decay. They also know that supernovas are powerful and prodigious generators of neutrinos and are essentially "neutrino bombs." $[13, \mathrm{p} 125]$ But the conventional astrophysical mechanisms turn out to generate energies many magnitudes below what has actually been detected with IceCube.

The prevailing thinking of astrophysicists is that the cosmic-sourced neutrinos come from some other kind of astrophysical phenomena in the distant universe, something considerably more powerful than a supernova. The favored contender is gamma-ray bursts. As reported in Scientific American, "Even brighter than regular supernovae, gammaray bursts are somewhat mysterious but probably occur during a special class of star collapse that involves very high mass stars under extreme conditions."[14, p61]

Or as astrophysics Professor Ray Jayawardhana stated, "... researchers think the particles may come from powerful jets shot out by monstrous black holes at the hearts of galaxies, or from incredible explosions known as gamma ray bursts (GRBs), which appear to be even more potent than supernovae."[13, p23]
The experts are remarkably close to resolving the mystery, as they are reasonably convinced of the ultra-energy source being the powerful jets and gamma ray bursts - phenomena associated with gravitationally collapsed objects. However, they still lack the specific generating mechanism.

The Mainspring, with its blueshifting and emitting processes, is that mechanism.

Mass formation formula ${ }^{3}$. When the high energy emissions of the Mainspring strike a target and generate mass, the process is in accordance with the conversion formula $m=E_{\text {frac! }}$ ! $c^{2}$, where $E_{\text {frac. }}$ is some portion (fraction) of the total energy of the incident photon or neutrino. The balance of the energy (the energy not converted to mass) goes into the kinetic energy of the products.

\subsection{Closing the Grand Loop}

Matter formation/creation, as an ongoing process, ultimately leads to the production of more Terminal neutron stars replacement generators of energy.

The matter distributed throughout the universe is, in a sense, a catalyst for the formation of more matter. The energy expelled from the Mainspring objects is largely converted to mass; and, naturally, this happens predominantly where mass is already present. Intergalactic particulate clouds will grow, replacing those that have collapsed into stars and protogalaxies. Galaxies will gain stars, replacing those that have collapsed. Galaxy clusters will acquire new Galaxies, replacing those that have been cannibalized. Galaxy clusters, thus supplied with new mass and energy, forever sustain their existence.

Mass formation also occurs, albeit at a far lower intensity, in the cosmic void regions. But because of the great vastness of those near-empty zones, the 'creation' rate can still be significant. Hence, it is probable (and the evidence supports this) that Voids will spawn various star systems - star clusters and dwarf galaxies and even medium-sized ellipticals. These will drift with the flow (the radially outward flow from the Void center) toward the nearest nodal galaxy cluster.

The regenerating system in a nutshell. Mainspringsourced energy is transformed to mass. The mass contributes to the formation of new stars. The mass also contributes to the collapse of existing stars. Finally, the mass contributes to the conversion of collapsed stars into end-state neutron stars (Terminal stars). Thus, new Mainspring objects are produced, replacing those that have been cannibalized and completing the conceptual circle of system components.

Through the workings of the basic laws of nature, Mainspring objects generate their own replacements.

\section{Discussion}

\subsection{The Aether 'Watershed' System}

The Mainspring mechanism is intimately tied to the subquantum level of existence. That is, it depends on all space (space as an empty container) being permeated by a

3 Derived from the relationship $E=m c^{2}$ attributed to Henri Poincaré (1854-1912). 
nonmaterial discretized aether. Without such medium, and an endless supply of it, there would be no "mechanism"; in fact, there would be no matter - no mass or energy particles whatsoever.

Given its importance, let us examine the aether system from a cosmic scale perspective - where the aether originates, how it flows, and where it ends up. The pattern, like a highlysymmetrical terrestrial watershed system, is shown in Figure 7. Although what is shown is the Universe's sub-physical domain, notice how the grand pattern coincides with the voids and galaxy clusters network of the physical domain. To clarify the point, the diagram only shows the nonphysical domain; but, from the systematic flow pattern, one can immediately identify the locations of the voids and galaxy clusters.

The emergence of aether (the nonmaterial medium) occurs in the Voids and the consumption/vanishment of aether occurs in the regions of matter concentrations. This 'watershed' system is more properly called a Voronoi cellular configuration.

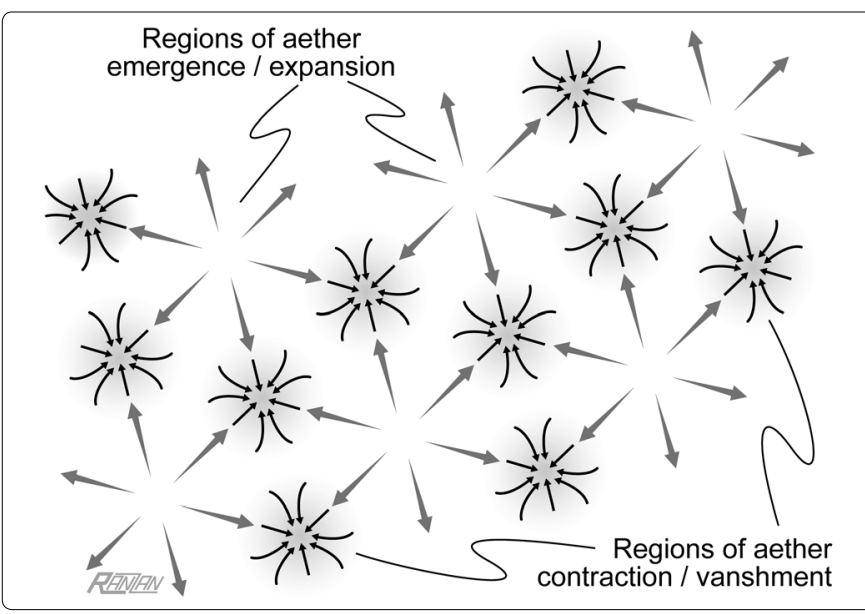

Figure 7. Cosmic picture (schematic cross-section) of the subphysical domain - the realm of the subquantum aether. Aether emerges within large empty regions - regions that are themselves part of a vast Voronoi 3-dimensional pattern- and undergoes contraction and 'consumption' at the Voronoi cell boundaries. Note, because of the effect the aether flow has on mass, the grand pattern necessarily coincides with the voids-and-clusters network of our cellular universe (DSSU).

It is not by coincidence that the flow pattern effectively maps out the two types of gravity regions. The aetheremergence regions are associated with divergent gravity, also known as cosmic antigravity. The aether-convergence regions are associated with conventional contractile gravity. In fact, it is the inhomogeneity of the flow of aether that is the veritable cause of gravity. Simply put, the very acceleration of the flow is what causes the gravitational effect [15] [2].

\subsection{Uniqueness of DSSU Aether}

The Mainspring mechanism represents the culmination of 20 years of successful development of DSSU theory; and the primary and essential factor responsible for the success lies with the unique nature of its universal space medium.

DSSU aether has these unique properties: (as can be seen in the chart (Figure 8) it consists of discrete entities; these entities are sub-physical (no mass, no energy); it has the dynamic ability to maintain a constant-count density. This dynamic ability manifests as aether emergence (expansion) and aether vanishment depending on stress conditions (tension, compression, shear). In the history of the development of the universal substrate, no other space medium has this combination of properties.

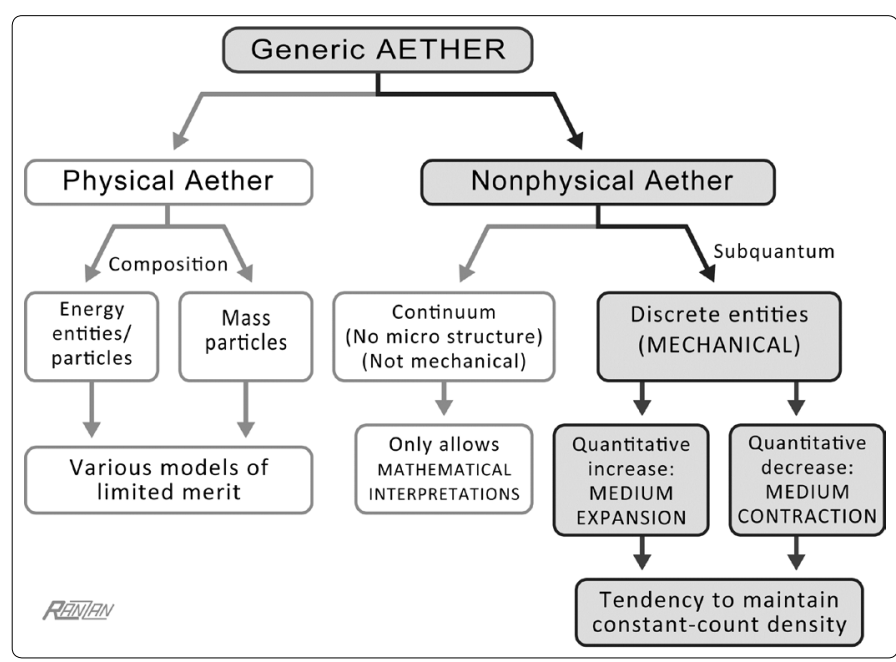

Figure 8. Categorization of aether. The "Physical" category leads to invalid theories (such as those of the $19^{\text {th }}$ century). The Nonphysical "continuum" category leads to mathematical constructs (notably Einstein's gravity theory). The Nonphysical "discretized" category leads to revolutionary developments in physics and cosmology. Traced by the dark-colored arrows connecting shaded blocks are the key defining characteristics of DSSU aether.

Notice the major division between Physical and Nonphysical types of aether. The Physical type has long been the most popular and continues to attract many researchers. Models built upon it, and this includes the popular quantum foam, invariably run up against insurmountable physical and philosophical problems. The historical record is littered with such failures.

Next, notice how the Nonphysical type is categorized as Continuous (necessarily nonmechanical) and as Discretized (mechanical). The Continuum type was the one used by Einstein; remember, he had said that the aether exists ("According to the general theory of relativity space without aether is unthinkable") but that it was not ponderable (no mass, no energy). Einstein's aether was purely a mathematical construct and championed by theoretical physicists.

The Discretized subcategory provides us, for the first time in history, with a subquantum mechanical aether featuring the dynamic ability to expand and to contract and to maintain a constant-count density.

\subsection{Continuous Versus Cyclical Creation}

The Mainspring mechanism works as a continuous regeneration of the universe. How does this approach compare with the ever popular Cyclical worldview?

There are, needless to say, many versions of the Cyclical type of universe but the general idea is a perennial loop of birth, death, and rebirth. The cosmos is somehow created or in some way creates itself, evolves over time, suffers total 
destruction, then reemerges -over and over in a replaymode existence, varying only as is necessary to comply with the entropy rule (the $2^{\text {nd }}$ law of thermodynamics). The Big Bang closed model is an exemplar; it bounces between big bang and big crunch. Here are its most serious problems.

- It has an irresolvable beginning-of-the-universe problem (the classic effect-without-a-cause dilemma). A multifacetted philosophical conundrum.

- Misinterpretation of an elementary phenomenon. It turns out the CMBR is not evidence of some primordial big bang. The CMBR is simply ultra-distant starlight something properly associated with the ambient temperature of the cosmos.

- There is no evidence of whole-universe expansion. It turns out that the cosmic redshift (discovered by Hubble and several others) is not caused by the recession of distant galaxies. With the discovery (in 2014) of the cosmic redshift mechanism of velocity differential propagation of light, the expanding-universe paradigm no longer has anything supporting it.

- There is no driving mechanism that is in any way compelling. This could also be expressed as a source-of-energy problem.

- It over-extrapolates a local theory of gravity (Einstein's general relativity).

- It withers under this hypothesis-destroying blow: At the final "crunch" stage, when all is being destroyed, and the "bounce" into a renewed creation is about to occur, the laws of physics go blank! The model breaks down.

In contrast, the continuous creation approach embodied in the Mainspring mechanism and used to sustain the Dynamic Steady State Universe has none of those problems.

\subsection{Thermodynamics}

There is no denying that both energy amplification and mass extinction are processes that violate the $1^{\text {st }}$ law of thermodynamics, the principle of matter conservation. An individual Terminal star may be a net generator/emitter of energy (radiation); or, it may be a net destroyer of energy (that is, more mass energy is eliminated than radiation is emitted). There is no cause-and-effect link between the two processes. One cannot, in an attempt to make the Mainspring object conform to the conservation law, impose a balance between the two processes, between ongoing energy emission on the one hand and mass extinction on the other.

On the cosmic scale of things, however, a balance is maintained - a self-balancing tendency between mass-andenergy production and mass-energy extinction. For more on this, see Section 4.3. in reference [5].

In conclusion, although local violation is inherent in the two processes, there is no overall violation of the first law of thermodynamics because the total energy of the universe, or more realistically any large cosmic region, remains always the same.
What about the $2^{\text {nd }}$ Law, the entropy rule? It simply does not apply to a Terminal star. It does not apply because a Terminal star is not an isolated system. Mass and radiation enter the system; and radiation is expelled from the system. So much for the Mainspring object as "the system." However, on the largest cosmic scale, the overall entropy remains constant, it never decreases; therefore, no violation of the $2^{\text {nd }}$ Law. In other words, the DSSU conforms to the relevant part of the entropy rule, which is that for the complete system, the large integrated entirety, the entropy, the measure of disorder, can never decrease.

For additional details, see the article published in Physics Essays journal (volume 34), Law of Physics 20th-Century Scientists Overlooked (Part 6): Cosmic-Scale Conservation of Energy [16].

\subsection{Funereal Views of Learned Academics}

It is a remarkable state of affairs. After almost a century since James Jeans revealed why and how the universe is dying, todays learned Academics still share his view, still believe the universe is irreversibly doomed. In the words of Leonard Susskind, "Modern-day scientists do predict that the universe -at least the universe as we know it - will come to an end. Every reasonable cosmological theory says so. When and how it will happen varies from one set of assumptions to another ..."[17] Why do they believe this? It is for the same reason that misled Jeans. Quoting physicist Paul Davies, "[E]ssentially all physical processes that we observe in the Universe are finite and nonrenewable.... The supply of material for new stars is limited."[18] In other words, the conviction continues to be that there is "no source of replenishment."

The modern end-of-the-world believers have considerably embellished Jeans' basic funereal scenario. Based on the false claim that the universe is expanding, they envision three possible fates.

- The world might end in fire. If the mass density in the universe is large enough to reverse the cosmic expansion, the final fate would be a big crunch, a super-hot implosion. ... The relativists think of such a universe as the distortion of spacetime into a mathematical " 3 -sphere" and call it a closed-and-bounded universe; and they think of its fate as a singularity.

- The world might end in deep-freeze. If the universe's mass density is significantly deficient (or the cosmological constant dominates), then the cosmic expansion would never end; the final fate would be a lifeless frozen dilution. ... The relativists think of it as the distortion of space into a mathematical hyperbolic artifact and call it an openand-unbounded universe; and they think of this fate as fairly likely.

- Another deep-freeze ending. If the mass density (and the cosmological constant) hold the universe on a knife-edge balance between the above two scenarios, then the universe would simply continue expanding forever. The relativists think of it as the absence of space distortion and call it a flat universe, a world with Euclidean space. 
There is no consensus on which it is to be. So, with new ideas prohibited, the final fate, at last report, remains a work in progress. But what is known, and is highly relevant to the root of the impasse, the 3 fates all have one thing in common. They all share the problem of being rooted in an uncritical interpretation of thermodynamic law.

In the absence of a regenerating process, the law of entropy becomes the ruling factor. The ruling principle is the advance of disorder. The deep structure of change is decay, corruption, and chaos -the unstemmable tide of thermodynamic chaos. Accordingly, philosophers delve into the pointlessness of the ultimate fate of the cosmos. They lament the bleakness, the lack of purpose, the unsustainability of the beating heart of the Universe.

They simply do not realize that thermodynamic laws are only local laws. Moreover, for the real universe there is no purpose in the ultimate fate -in fact, there is no ultimate fate. The Universe simply IS. Beyond its being, it has no ultimate fate - none whatsoever.

\section{Summary and Conclusion}

\subsection{Overview of Mainspring}

Overview of the Mainspring as an input-output system is depicted in Figure 9. On the input side, the system feeds on a steady inflow of matter and radiation, and the occasional spectacular gravitational ingestion. On the output side, there is the expulsion of energy (photons and neutrinos) into the external realm; there is also the expulsion of any excess mass into the 'realm' of non-existence.

Overview of the Mainspring as a functional system:

- Ejects energy carriers, photons and neutrinos, through polar portals;

- Converts some of the input mass into pure energy;

- Amplifies the energy trapped within the surface layer;

- Destroys any and all excess mass.

Also, the subquantum medium plays a crucial role (as was discussed in Section 5.1).

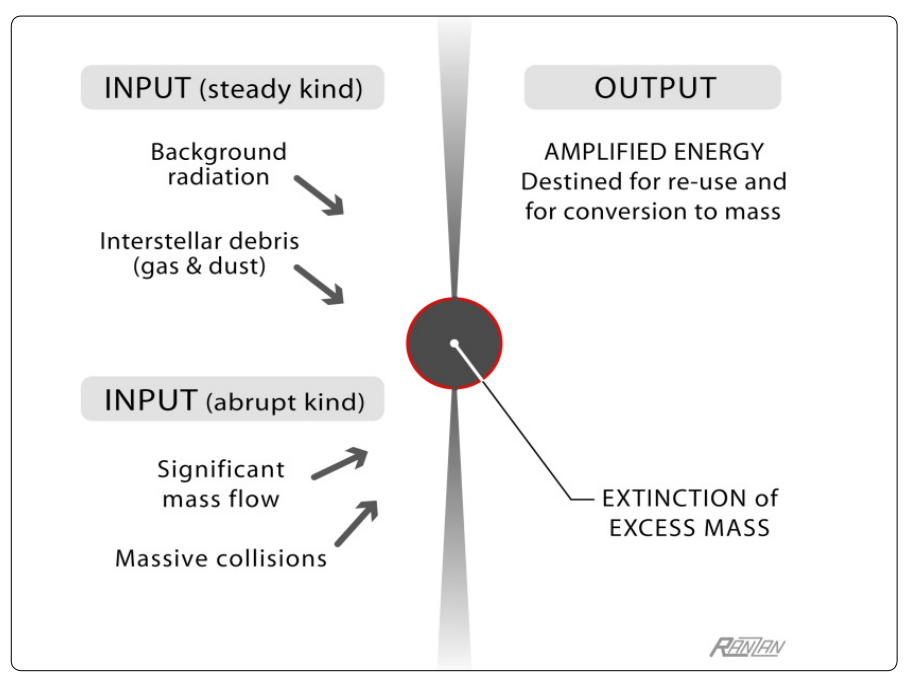

Figure 9. Mainspring viewed as an input-output system - with all manner of radiation and mass coming in and amplified radiation going out. The essential functional parts of the system are (i) Ejection of energy carriers, photons and neutrinos; (ii) Conversion of some of the infalling mass into pure energy; (iii) Amplification of the energy trapped within the surface layer; (iv) Destruction/ vanishment of any excess mass. (Schematic cross-section)

There is a remarkable elegance and simplicity in the way the system works - the way the several processes fit together. Notice the perpetual nature of the processes. Notice the limitless aspect of the supply of regenerated energy. Notice the remarkable inevitability in the way the several elements of the system are combined. Inherently inevitable, pleasingly uncomplicated, metaphysically profound; these are the conclusions that follow when the system is evaluated in the light of the underlying principles.

The following are the underlying principles upon which the Mainspring depends, directly or indirectly.

\section{Universal principles:}

1. Primary process principle: Mentioned here but not discussed. Also called the Essence process in [19, p314].

2. Principle of physicality: All mass and energy particles (and electromagnetic waves) exist by virtue of aether excitation and accompanying annihilation/vanishment of aether. (Such particles, if deprived of aether, must cease to exist.) This principle is rooted in the way photons propagate. Whether free (as radiation) or confined (as mass), photons propagate as an excitation of aether, an excitation that actually destroys aether units, leaving 'holes' in the medium, 'holes' which are immediately filled by the surrounding aether [19, Figures 17 and 18].

\section{Principles relevant to the Mainspring object:}

3. Principle of mass extinction by aether deprivation: It follows directly from the principle of physicality. While the presence of aether sustains the existence of mass, the absence of aether precludes its existence. Principles \#2 and \#3 are like two faces of the same coin. [5]

4. Principle of energy generation by velocity differential propagation of radiation: Simply called the Blueshifting mechanism. This is Nature's fundamental energy amplification process - the prime mover of the Mainspring. The proof is published in [20].

5. Principle of energy ejection: Refers to the mechanism that maintains the polar escape portals. [20]

\section{Principles relevant to the formation of new Mainspring} objects:

6. Principle of energy-to-mass conversion: Energy is transformable to mass in accordance with experimentally validated rules of particle physics.

7. Principle of end-state gravitational collapse: Although there may be several ways the 'end state' comes about, the concern here is only with the final state - the Terminal star. There are two requirements involved [8].

- The existence of a specific mass-density limit; a density that Nature cannot exceed. 
- Aether inflow at the structure's surface cannot exceed lightspeed; just a basic special-relativity requirement.

Justifiable conclusion. The operation, the functionality, of the Mainspring object presents science with a supreme advantage. Unlike the situation plaguing $20^{\text {th }}$-century blackhole theory, nowhere is there a breakdown of the underlying physics.

\subsection{Quick Summary Flowchart}

The Universe is ruled entirely by processes. Behind the apparently real and imagined forces, behind the various manifestations of energy, there are only processes.

The processes of the physical realm are diagrammed in Figure 10. Also, there are the processes of the sub-physical domain. They were briefly discussed in connection with Figure 7; they consist of aether emergence (expansion) and aether self-vanishment (contraction) and aether excitationconsumption (by matter).

In addition to summarizing the processes of the physical realm, the flowchart (Figure 10) is an attempt to show how the Mainspring interacts with the external World, how it relates to the rest of the Universe. As an aid in interpreting the flowchart, remember this difference between the Mainspring system and the surrounding cosmic system: The processes occurring within the first do not conform to thermodynamic laws, while all the processes (all the various kinds of energy transformations) taking place within the latter do so in accordance with the conventional laws of thermodynamics.

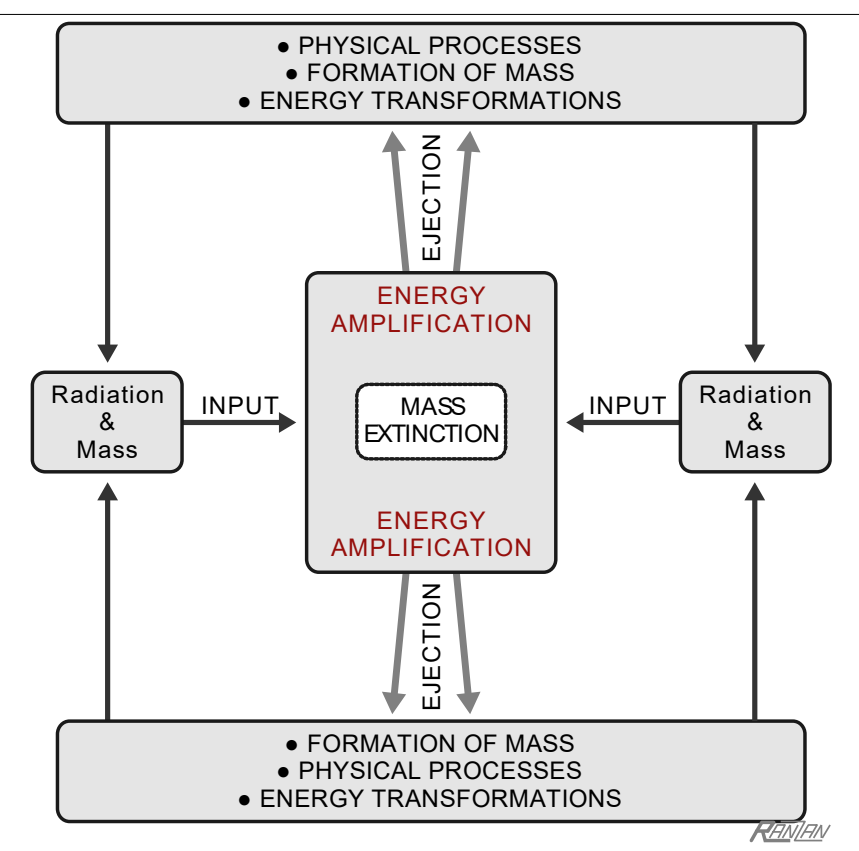

Figure 10. Simplified flowchart of the processes (upper case terms) that constitute the Universe. The layout is designed to show how the Mainspring object (central block) fits into the larger external system (surrounding blocks). The "EJECTION" beams convey the vital Primary radiation. Note, the operation of the system is not cyclical; rather, it is continuous.

\section{Glossary of the terms/labels used in Figure 10:}

Energy amplification: energy generation by velocity differential propagation of radiation. This is the Blueshifting process.
Mass extinction: Refers to the loss of mass by aether deprivation. It follows from the fact that mass cannot exist when it is deprived of aether (see the principle of physicality, above).

Ejection: Refers to the outbound propagation of photons and neutrinos - passing through the polar escape portals. This is what constitutes the Terminal star's emission beams and is what powers astronomically observed astrophysical jets.

Formation of mass: The conversion of gamma photons to subatomic mass particles. The conversion of ultra-high energy neutrinos to protons, neutrons, mesons, etc.

Physical processes: Gravitational aggregation. The evolution of stars and planets and life.

Energy transformations: Energy reactions/conversions in accordance with the Law of Conservation of Energy.

Radiation (INPUT): Starlight of all wavelengths, including its most ubiquitous form, the microwave background radiation.

Mass (INPUT): Generally refers to interstellar dust and gas; but may include objects of any size or content —even another Mainspring object.

Be reminded, the overall system with its countless Terminal stars interacting with the vast Cosmos is not to be thought of as a cyclical sequence. Rather, it is to be viewed as a set of ongoing processes of identifiable definable activities that take place all the time (all in accordance with the basic laws of physics and the seven principles cited earlier) - steady state and without end.

\subsection{Final Words}

What drives the Cosmos? What lies beneath its forces? What sustains it energy? ... The answer is processes. The Universe is entirely maintained by the processes presented in the Flowchart and the dynamics of the sub-physical domain. The profound conclusion is that the processes of the Mainspring mechanism and those of the sub-physical domain sustain the Universe perpetually - it will continue in its present state (steady state) forever.

What prevents the universe from running down? What keeps the universe alive?

The profound answer to James Jeans' query is this: The universe does have a source of replenishment. Our world is prevented from running down by the energy generated through its Mainspring objects. The universe is alive in the sense that its Mainspring objects generate their own replacements. It is kept 'alive' perpetually.

\section{References}

1. Jeans J. Chapter VI, Beginnings and Endings, The Universe Around Us. 3rd ed. Cambridge University Press, London; 1933.

2. Ranzan C. The Nature of Gravity - How one factor unifies gravity's convergent, divergent, vortex, and wave effects. International Journal of Astrophysics and Space Science. 2018; 6(5): 73-92. doi: 10.11648/j. ijass.20180605.11

3. Ranzan C. Cosmic Redshift in the Nonexpanding Cellular Universe: VelocityDifferential Theory of Cosmic Redshift. American Journal of Astronomy \& Astrophysics. 2014; 2(5): 47-60. doi: 10.11648/j.ajaa.20140205.11 
4. Ranzan C. Natural Mechanism for the Generation and Emission of Extreme Energy Particles. Physics Essays. 2018; 31(3): 358-376. doi: 10.4006/0836-1398-31.3.358

5. Ranzan C. Law of Physics 20th-Century Scientists Overlooked (Part 4): Mass Extinction by Aether Deprivation. Journal of High Energy Physics, Gravitation and Cosmology. 2021; 7(1): 191-209. doi: 10.4236/jhepgc.2021.71010

6. Ranzan C. Law of Physics 20th-Century Scientists Overlooked (Part 5): Centrifugal Effect Negation. Applied Physics Research. 2021; 13(2) 13-34.

7. Einstein A. Äther und Relativitätstheorie. Lecture Presented on 5th May, 1920 in the University of Leyden (Berlin: Springer). 1920. English translation is posted at http://www.tu-harburg.de/rzt/rzt/it/Ether.html

8. Ranzan C. The Nature of Gravitational Collapse. American Journal of Astronomy and Astrophysics. 2016; 4(2): 15-33. doi: 10.11648/j. ajaa.20160402.11

9. Pollock S. Great Ideas of Classical Physics. Course Guidebook, Guidebook p45. The Teaching Co., Virginia; 2006.

10. Ranzan C. Law of Physics 20th-Century Scientists Overlooked (Part 1): The Velocity Differential Propagation of Light. Physics Essays. 2020; 33(2): 163-174. doi: 10.4006/0836-1398-33.2.163

11. Encyclopedia Britannica. Edition.15; V.25. p856.
12. Wilczek F. The Lightness of Being. Basic Books, New York, N. Y; 2008.

13. Jayawardhana R. Neutrino Hunters. Harper Collins, Toronto; 2013.

14. Halsen F. Neutrons at the Ends of the Earth. Scientific American; P60. October 2015

15. Cahill R.T. Absolute Motion and Gravitational Effects. Apeiron. 2004; 11(1)

16. Ranzan C. Law of Physics 20th-Century Scientists Overlooked (Part 6): Cosmic-Scale Conservation of Energy. Physics Essays. 2021; 34(3): 332-340. doi:10.4006/0836-1398-34.3.332

17. Susskind L. The Cosmic Landscape, String Theory and the Illusion of Intelligent Design. p142. Back Bay Books. Little, Brown and Co.; New York; 2006.

18. Davies P., Gribbin J. The Matter Myth. p123. Simon \& Schuster, Touchstone, New York; 1992.

19. Ranzan C. The Dynamic Steady State Universe. Physics Essays. 2014; 27(2): 286-315. doi: 10.4006/0836-1398-27.2.286

20. Ranzan C. Law of Physics 20th-Century Scientists Overlooked (Part 2): Energy Generation via Velocity Differential Blueshift. Physics Essays. 2020; 33(3): 289-298. doi: 10.4006/0836-1398-33.3.289 